\title{
Caracterização anatômica macroscópica do lenho de árvores de espécies de canela, Ocotea e Nectandra (Lauraceae), ocorrentes no estado de Santa Catarina
}

\author{
Felipe Bernardino Guimarães $1,3 *$ \\ Mário Tomazello Filho ${ }^{2}$ \\ Ana Claudia Rodrigues ${ }^{3}$ \\ ${ }^{1}$ Instituto Brasileiro do Meio Ambiente e Recursos Naturais Renováveis - IBAMA/SC \\ Rua Conselheiro Mafra, 784, Centro, CEP 88010-102, Florianópolis - SC, Brasil \\ ${ }^{2}$ Universidade de São Paulo, Escola Superior de Agricultura Luiz de Queiroz (ESALQ/USP) \\ Piracicaba - SP, Brasil \\ ${ }^{3}$ Universidade Federal de Santa Catarina \\ Programa de Pós-Graduação em Biologia de Fungos, Algas e Plantas \\ Florianópolis - SC, Brasil \\ * Autor para correspondência \\ felipebiolguimaraes@hotmail.com
}

Submetido em 25/11/2016

Aceito para publicação em 25/04/2017

\section{Resumo}

As árvores conhecidas popularmente por "canela", pertencentes aos gêneros Ocotea e Nectandra (Lauraceae), são de difícil separação, tanto em nível de gênero como de espécie, pela anatomia do seu lenho. O presente trabalho tem como objetivo descrever os caracteres anatômicos macroscópicos e organolépticos do lenho e sua aplicação na identificação dos gêneros e das espécies desta família. Foram coletados discos do lenho à altura do peito $(1,30 \mathrm{~m})$ do tronco das árvores de Nectandra megapotamica, $N$. lanceolata, Ocotea porosa, O. puberula, O. pulchella e Ocotea sp. ocorrentes em quatro municípios do interior do estado de Santa Catarina. As características anatômicas macroscópicas e organolépticas do lenho foram descritas de acordo com as normas usuais para estudos em anatomia da madeira, cujos resultados indicam a possibilidade de separação de Nectandra e de Ocotea, tais como visibilidade do parênquima axial, arranjo diagonal dos vasos, cor e cheiro do lenho. Apresenta-se, ainda, uma chave dicotômica que permite a separação das árvores das espécies de Lauráceas estudadas. No trabalho, conclui-se que as seis espécies analisadas podem ser identificadas pela estrutura anatômica observada macroscopicamente e pelas propriedades organolépticas do seu lenho, não sendo encontrados, no entanto, caracteres que propiciem a distinção dos gêneros Ocotea e Nectandra entre si.

Palavras-chave: Anatomia do lenho; Caracterização macroscópica; Identificação de madeiras; Propriedades organolépticas

\section{Abstract}

Wood anatomy of trees of "canela" species (Ocotea and Nectandra, Lauraceae) from Santa Catarina, Brazil. The trees known as "canela" belong to the genera Ocotea and Nectandra (Lauraceae) and are difficult 
to identify at the genus and species level based on their wood. The present work describes anatomical and organoleptic characteristics of the wood of Ocotea and Nectandra species that can be used for identification. Wood discs were collected at breast height (BH $1.30 \mathrm{~m}$ ) from trunks of Nectandra megapotamica, N. lanceolata, Ocotea porosa, O. puberula, O. pulchella and Ocotea sp., in four municipalities in the interior of Santa Catarina State. The macroscopic anatomy and organoleptic characteristics were described based on standard techniques used in wood anatomy studies. The results show that the species of Nectandra and Ocotea can be separated by their axial parenchyma distinctness, diagonal arrangement of vessels, wood color and odor. A dichotomous key to the studied species of Lauraceae is also provided. The present work found that it was possible to identify the species studied using macroscopic anatomy and organoleptic properties of the wood; however, it was not possible to distinguish Ocotea from Nectandra.

Key words: Macroscopic characterization; Organoleptic properties; Wood anatomy; Wood identification

\section{Introdução}

A estrutura anatômica do xilema secundário da família Lauraceae aparenta ser, em termos filogenéticos, transicional a avançada, mais do que primitiva (STERN, 1954; ROHWER, 1993). A uniformidade dos caracteres anatômicos do xilema secundário nesta família é bastante evidente (SOLEREDER, 1908; STERN, 1954; KOSTERMANS, 1957; RICHTER, 1981; 1987), o que facilita inclusive a identificação de fósseis deste grupo (TUPPER, 1927). Entre os caracteres anatômicos mais significativos do xilema secundário que caracterizam a família Lauraceae podem ser citados: a porosidade difusa; os vasos nunca exclusivamente solitários, arredondados ou ovais; as pontoações intervasculares sempre alternas; a presença de parênquima paratraqueal; as fibras com pontoações localizadas exclusivamente nas paredes radiais; os raios estreitos, geralmente de 1-4 células de largura; a presença de células oleíferas/mucilaginosas na maioria das espécies associadas aos parênquimas axial e/ou radial (RICHTER, 1981; 1987; 1990; METCALFE; CHALK, 1985; CALLADO; COSTA, 1997; LEÓN; ESPINOZA DE PERNÍA 2000a; 2000b; OLIVEIRA et al., 2001). Padrões e similaridades anatômicas também têm sido identificados para gêneros (RICHTER, 1981; ROHWER, 1991), mas incertezas taxonômicas de alguns grupos, especialmente Ocotea e Nectandra, claramente indicados como parafiléticos, se refletem também na dificuldade de observação de padrões anatômicos nos caracteres da madeira e da casca. Alguns gêneros possuem características anatômicas bastante específicas, que os distinguem dos demais gêneros da família, p.ex. anéis porosos somente em espécies de Sassafras (RICHTER, 1987), e raios estratificados em espécies de Aspidostemon e algumas espécies de Beilschmiedia e Potameia (ROHWER, 1993). No entanto, a grande maioria dos gêneros dentro da família compartilha caracteres anatômicos do xilema secundário de forma bastante homogênea, tornando difícil e complexa a identificação tanto ao nível genérico quanto específico (CASTIGLIONI, 1962; RICHTER, 1981; 1987).

Alheios aos conflitos taxonômicas da família Lauraceae encontram-se os atores envolvidos na cadeia produtiva das espécies de canela exploradas comercialmente: extratores, madeireiros, comerciantes, consumidores e agentes da lei, sendo estes últimos exigidos a identificarem as espécies envolvidas em crimes ambientais de forma rápida e com as ferramentas disponíveis em campo: estilete e lupa conta-fios. Embora ferramentas que facilitem a identificação de madeiras estejam sendo desenvolvidas para auxiliar profissionais que trabalhem nesta área na identificação de espécies, como a Chave Interativa para Identificação de Madeiras Comerciais do Brasil (CORADIN et al., 2009), poucos são os dados disponíveis acerca das espécies de Lauraceae. Pretendemos, assim, contribuir para o conhecimento da anatomia do xilema secundário das árvores de algumas espécies dos gêneros Ocotea e Nectandra conhecidas sob a denominação de canela, ocorrentes no estado de Santa Catarina.

\section{Material e Métodos}

As áreas de coleta situam-se no oeste, meio-oeste e planalto serrano catarinense, possuem altitudes, 
temperaturas médias anuais e pluviosidade média anual muito similares: Passos Maia $-1050 \mathrm{~m}$ altitude, $\mathrm{T}^{\circ}$ média $16,6^{\circ} \mathrm{C}, 190 \mathrm{~mm}$ precipitação média anual; Tangará $600 \mathrm{~m}$ altitude, $\mathrm{T}^{\circ}$ média $17,3^{\circ} \mathrm{C}, 155 \mathrm{~mm}$ precipitação média anual; Abdon Batista $-700 \mathrm{~m}$ altitude, $\mathrm{T}^{\mathrm{o}}$ média $17,5^{\circ} \mathrm{C}, 153 \mathrm{~mm}$ precipitação média anual e Concórdia $-750 \mathrm{~m}$ altitude, $\mathrm{T}^{\circ}$ média $17,2^{\circ} \mathrm{C}, 168 \mathrm{~mm}$ precipitação média anual.

As coletas foram realizadas em áreas de supressão vegetal para instalação da Pequena Central Hidrelétrica (PCH) no Rio Chapecó (Passos Maia $26^{\circ} 42^{\prime} 06,5^{\prime}$ 'S $/ 51^{\circ} 55^{\prime} 12,6^{\prime}$ 'W) e PCH de Salto Góes, no

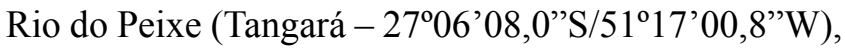
da Usina Hidrelétrica Garibaldi (UHE Garibaldi), no Rio

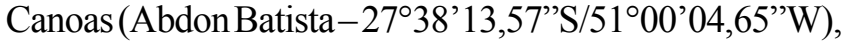
e do Loteamento Frei Lency II (Concórdia - 27 $14^{\prime} 03$, 5”S/51 ${ }^{\circ} 57^{\prime} 48,93$ ”W). As áreas possuíam autorização de supressão (AUTEX), emitidas pela FATMA, e as coletas de madeira e dos ramos das árvores foram realizadas em acompanhamento aos trabalhos das equipes de supressão em campo. Foram coletados três discos do lenho à altura do peito $(1,30 \mathrm{~m})$ de árvores de canela das espécies Nectandra lanceolata Nees, N. megapotamica (Spreng.) Mez, Ocotea puberula (Rich.) Nees, O. pulchella (Nees) Mez, O. porosa (Nees \& C. Mart.) Barroso e Ocotea sp. As alturas e os diâmetros médios das árvores bem como o número de indivíduos coletados em cada área estão resumidos na Tabela 1 .

As exsicatas foram depositadas no herbário FLOR e encaminhadas para dois especialistas para identificação. As cunhas de madeira foram depositadas na Xiloteca do Herbário Flor e na Xiloteca Calvino Manieri (BCTw) no Instituto de Pesquisas Tecnológicas em São Paulo (Tabela 2).

TABELA 1: Relação das espécies coletadas, locais de coleta, número de indivíduos coletados, DAP médio e altura média das árvores coletadas.

\begin{tabular}{lcccc}
\hline \multicolumn{1}{c}{ Espécies } & Locais de coleta & № amostral & DAP Médio & Altura Média \\
\hline \multirow{2}{*}{ Nectandra lanceolata Nees } & Abdon Batista & 07 & 12,479 & 10,143 \\
& Concórdia & 07 & 26,379 & 15,429 \\
& Tangará & 07 & 21,864 & 14,714 \\
\hline \multirow{2}{*}{ Nectandra megapotamica (Spreng.) Mez } & Abdon Batista & 08 & 13,850 & 11,500 \\
& Concórdia & 06 & 19,542 & 12,833 \\
& Tangará & 07 & 17,686 & 12,429 \\
\hline Ocotea porosa (Nees \& C. Mart.) Barroso & Passos Maia & 01 & 56,400 & 21,000 \\
\hline \multirow{2}{*}{ Ocotea puberula (Rich.) Nees } & Abdon Batista & 07 & 16,943 & 13,143 \\
& Concórdia & 07 & 25,862 & 16,769 \\
\hline \multirow{2}{*}{ Ocotea pulchella (Nees) Mez } & Tangará & 07 & 20,500 & 15,429 \\
\hline Ocotea sp. & Abdon Batista & 01 & 32,800 & 15,000 \\
\hline
\end{tabular}


TABELA 2: Relação das espécies coletadas, número de registro das exsicatas no Herbário FLOR e no de registro das amostras de madeira na xiloteca Dr. Calvino Manieri (BCTw) no Instituto de Pesquisas Tecnológicas de São Paulo.

\begin{tabular}{|c|c|c|}
\hline Espécie & FLOR & BCTw \\
\hline Nectandra lanceolata Nees & $\begin{array}{l}\text { 39755; 39765; 39767; 39771; 39775; 39780; } \\
\text { 39781; 39587; 39598; 39600; 39604; 39610; } \\
\text { 39611; 39544; 39546; 39545; 39671; 39672; } \\
\text { 39696; 39697; 39700. }\end{array}$ & $\begin{array}{l}\text { 7389; 7392; 7394; 7402; 7408; 7413; 7414; } \\
\text { 7475; 7550; 7554; 7595; 7606; 7611; 9159; } \\
9160 ; 9161 ; 7364 ; 7361 ; 7294 ; 7289 ; 7283 .\end{array}$ \\
\hline $\begin{array}{l}\text { Nectandra megapotamica } \\
\text { (Spreng.) Mez }\end{array}$ & $\begin{array}{l}\text { 39743; 39744; 39745; 39749; 39750; 39754; } \\
\text { 39777; 39789; 39597; 39601; 39602; 39603; } \\
\text { 39606; 39607; 39547; 39540; 39539; 39675; } \\
\text { 39685; 39686; 39695. }\end{array}$ & $\begin{array}{l}\text { 7517; 7518; 7519; 7380; 7382; 7387; 7410; } \\
\text { 7422; 7548; 7567; 7573; 7575; 7597; 7601; } \\
9164 ; 9166 ; 9168 ; 7357 ; 7324 ; 7323 ; 7298 .\end{array}$ \\
\hline $\begin{array}{l}\text { Ocotea porosa (Nees \& C. } \\
\text { Mart.) Barroso }\end{array}$ & 39550 & 9157 \\
\hline $\begin{array}{l}\text { Ocotea puberula (Rich.) } \\
\text { Nees }\end{array}$ & $\begin{array}{l}\text { 39711; 39736; 39740; 39746; 39751; 39752; } \\
\text { 39753; 39588; 39590; 39592; 39599; 39608; } \\
\text { 39613; 39614; 39543; 39541; 39542; 39647; } \\
\text { 39674; 39679; 39690. }\end{array}$ & $\begin{array}{l}7525 ; 7504 ; 7513 ; 7520 ; 7383 ; 7384 ; 7385 \\
7476 ; 7485 ; 7488 ; 7553 ; 7603 ; 7623 ; 7624 \\
9158 ; 9162 ; 9163 ; 7371 ; 7359 ; 7349 ; 7314 .\end{array}$ \\
\hline $\begin{array}{l}\text { Ocotea pulchella (Nees) } \\
\text { Mez }\end{array}$ & $38979 ; 38984 ; 39548 ; 39549 ; 39797$ & 9155; 9156; 9154; 9153; 7435; \\
\hline Ocotea sp. & 39605 & 7596 \\
\hline
\end{tabular}

Os caracteres macroscópicos do lenho foram analisados de acordo com o proposto por Coradin e Muñiz (1991) e Coradin et al. (2009). As análises do lenho foram feitas a olho nu ou sob lupa de 10x e as fotografias obtidas com microscópio estereoscópio Leica EZ4D e software LAS 3.0. Realizou-se ainda o teste químico com Cromazurol-S (KUKACHKA; MILLER, 1980) para detecção de alumínio no lenho.

\section{Resultados}

\section{Nectandra lanceolata - Canela-amarela (Figura $1 \mathrm{~A}-\mathrm{H})$}

Características gerais: Cerne/alburno indistintos pela cor; cerne amarelado, com alteração de cor por foto-oxidação, mudando para amarronzado-acinzentado (Figura 1B-C); anéis de crescimento distintos, individualizados por zonas fibrosas mais escuras (Figura 1D); madeira com brilho nas superfícies transversal e longitudinais (Figura 1G-H); sem cheiro perceptível; moderadamente dura ao corte transversal manual; grã revessa; textura média; figura presente, em faixas causadas pelas camadas de crescimento (podendo formar "V" ou "U"). Poros: Presentes, visíveis apenas com lente de 10x, pequenos ou médios, de distribuição difusa, frequência média, predominantemente solitários, eventualmente múltiplos radiais com menos de quatro vasos, eventualmente dispostos em arranjo diagonal; formato circular a oval; placas de perfuração não observadas mesmo com lente de 10x; parte dos vasos obstruídos por tilos, poucos (Figura 1E-F). Parênquima axial: distinto sob lente de $10 \mathrm{x}$, com dificuldade, paratraqueal escasso ou vasicêntrico (Figura 1E-F). Raios: Observados apenas com lente de 10x nas superfícies transversal (Figura 1E) e longitudinal tangencial (Figura 1G), visíveis a olho nu e bem contrastados na superfície longitudinal radial (Figura $1 \mathrm{H})$, finos, baixos, pouco frequentes. Estruturas estratificadas: Ausentes. Estruturas secretoras: Ausentes. Variantes cambiais: Não observadas. Testes químicos: Teste de Cromazurol negativo. 
FIGURA 1: Nectandra lanceolata. A. Ramo da árvore; B. Disco do tronco recém-cortado; C. Disco do tronco lixado; D. Face transversal mostrando anéis de crescimento marcados por zonas fibrosas; E. Secção transversal (10x); F. Secção transversal (20x); G. Secção longitudinal tangencial (10x); H. Secção longitudinal radial da madeira (10x). Escalas: A (6 cm); B (10 cm); C (7 cm); D $(2 \mathrm{~cm})$; E-H $(1 \mathrm{~mm})$.
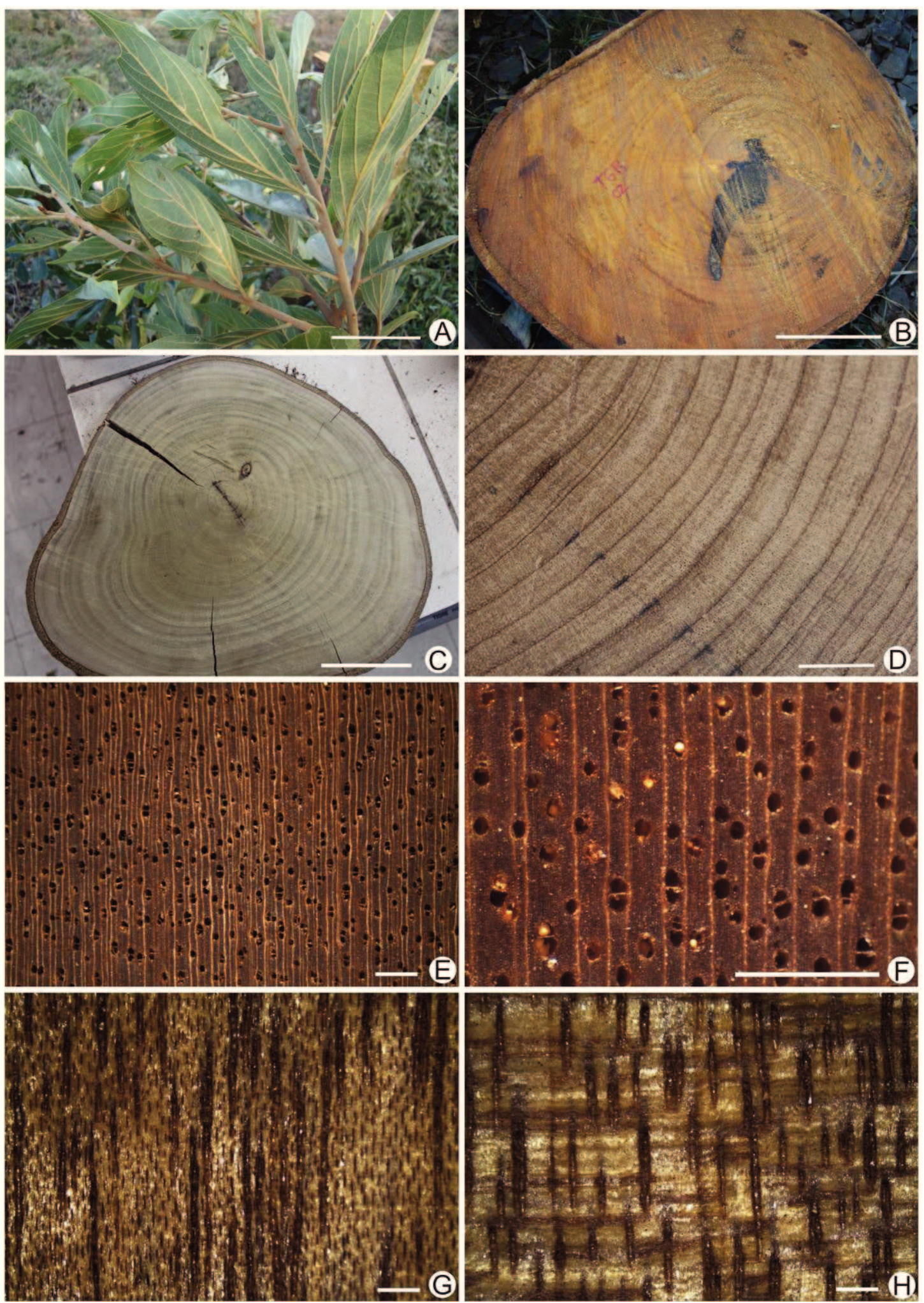
Nectandra megapotamica - Canela-amarela (Figura 2A-H)

Características gerais: Cerne/alburno pouco distintos pela cor; cerne amarelado, com alteração de cor por foto-oxidação, mudando para amarronzadooliváceo (Figura 2B-C); anéis de crescimento distintos, individualizados por zonas fibrosas mais escuras (Figura 2D); madeira com brilho nas superfícies transversal e longitudinais (Figura 2G-H); com cheiro perceptível, desagradável, que desaparece com a secagem da madeira, retornando se ela for molhada; moderadamente dura ao corte transversal manual; grã revessa; textura média; figura presente, em faixas causadas pelas camadas de crescimento (podendo formar "V" ou "U"). Poros: Presentes, visíveis apenas com lente de 10x, pequenos ou médios, de distribuição difusa, frequência média; predominantemente solitários, eventualmente múltiplos radiais com menos de 4 vasos; eventualmente dispostos em arranjo diagonal; de formato circular a oval; placas de perfuração não observadas mesmo com lente de 10x; parte dos vasos obstruídos por tilos (Figura 2E-F). Parênquima axial: pouco distinto sob lente de 10x, paratraqueal escasso ou vasicêntrico (Figura 2E-F). Raios: Observados apenas com lente de 10x nas superfícies transversal (Figura 2E) e longitudinal tangencial (Figura 2G), visíveis a olho nu e bem contrastados na superfície longitudinal radial (Figura $2 \mathrm{H})$, finos, baixos, pouco frequentes. Estruturas estratificadas: Ausentes. Estruturas secretoras: Ausentes. Variantes cambiais: Não observadas. Testes químicos: Teste de Cromazurol negativo.

\section{Ocotea porosa - Imbuia (Figura 3A-G)}

Características gerais: Cerne/alburno distintos pela cor; cerne marrom-amarelado, com alteração acentuada de cor por foto-oxidação, mudando para amarronzado-oliváceo (Figura 3B); anéis de crescimento distintos, individualizados por zonas fibrosas mais escuras (Figura 3C). Madeira com brilho nas superfícies transversal e longitudinais (Figura 3F-G), com cheiro perceptível, agradável, moderadamente dura ao corte transversal manual; grã direita a revessa; textura média, figura presente em faixas causadas pelas camadas de crescimento (podendo formar "V" ou "U").
Poros: Presentes, visíveis apenas com lente de 10x, pequenos, de distribuição difusa, frequência média, predominantemente solitários, eventualmente múltiplos radiais com menos de 4 elementos, sem padrão definido de disposição; de formato circular a oval; placas de perfuração não observadas mesmo com lente de 10x; parte dos vasos obstruídos por tilos (Figura 3D-E). Parênquima axial: Não observado, mesmo sob lente de 10x (Figura 3D). Raios: Observados apenas com lente de 10x nas superfícies transversal (Figura 3D) e longitudinal tangencial (Figura 3F), bem contrastados na superfície radial (Figura 3G), finos, baixos, pouco frequentes. Estruturas estratificadas: Ausentes. Estruturas secretoras: Ausentes. Variantes cambiais: Não observadas. Testes químicos: Teste de Cromazurol negativo.

\section{Ocotea puberula - Canela-guaicá (Figura 4A-H)}

Características gerais: Cerne/alburno indistintos pela cor; cerne branco-amarelado-acinzentado, com alteração acentuada de cor por foto-oxidação, mudando para amarronzado-acinzentado (Figura 4B-C); anéis de crescimento distintos, individualizados por zonas fibrosas mais escuras (Figura 4D); madeira com brilho nas superfícies longitudinais (Figura 4G-H); sem cheiro perceptível; macia ao corte transversal manual; grã direita a revessa; textura média; figura presente, em faixas causadas pelas camadas de crescimento (podendo formar "V" ou "U"). Poros: Presentes, visíveis apenas com lente de 10x, pequenos ou médios, de distribuição difusa; frequência média; solitários, frequentemente geminados e múltiplos radiais com menos de 4 vasos, ou mais raramente com até 7 elementos, dispostos em padrão não definido, de formato circular a oval; placas de perfuração não observadas mesmo com lente de 10x.; vasos eventualmente obstruídos por tilos (Figura 4E-F). Parênquima axial: Não observado, mesmo sob lente de 10x (Figura 4E). Raios: Observados apenas com lente de 10x nas superfícies transversal e longitudinal tangencial (Figura 4G), visíveis a olho nu e bem contrastados na superfície longitudinal radial (Figura 4H), finos, baixos, pouco frequentes. Estruturas estratificadas: Ausentes. Estruturas secretoras: Ausentes. Variantes cambiais: Não observadas. Testes químicos: Teste de Cromazurol negativo. 
FIGURA 2: Nectandra megapotamica. A. Ramo da árvore; B. Disco do tronco recém-cortado; C. Disco do tronco lixado; D. Face transversal com anéis de crescimento bem definidos; E. Secção transversal (10x); F. Secção transversal (20x); G. Secção longitudinal tangencial (10x); H. Secção longitudinal radial (10x). Escalas: A-C (6 cm); D (3 cm); E-H (1 mm).
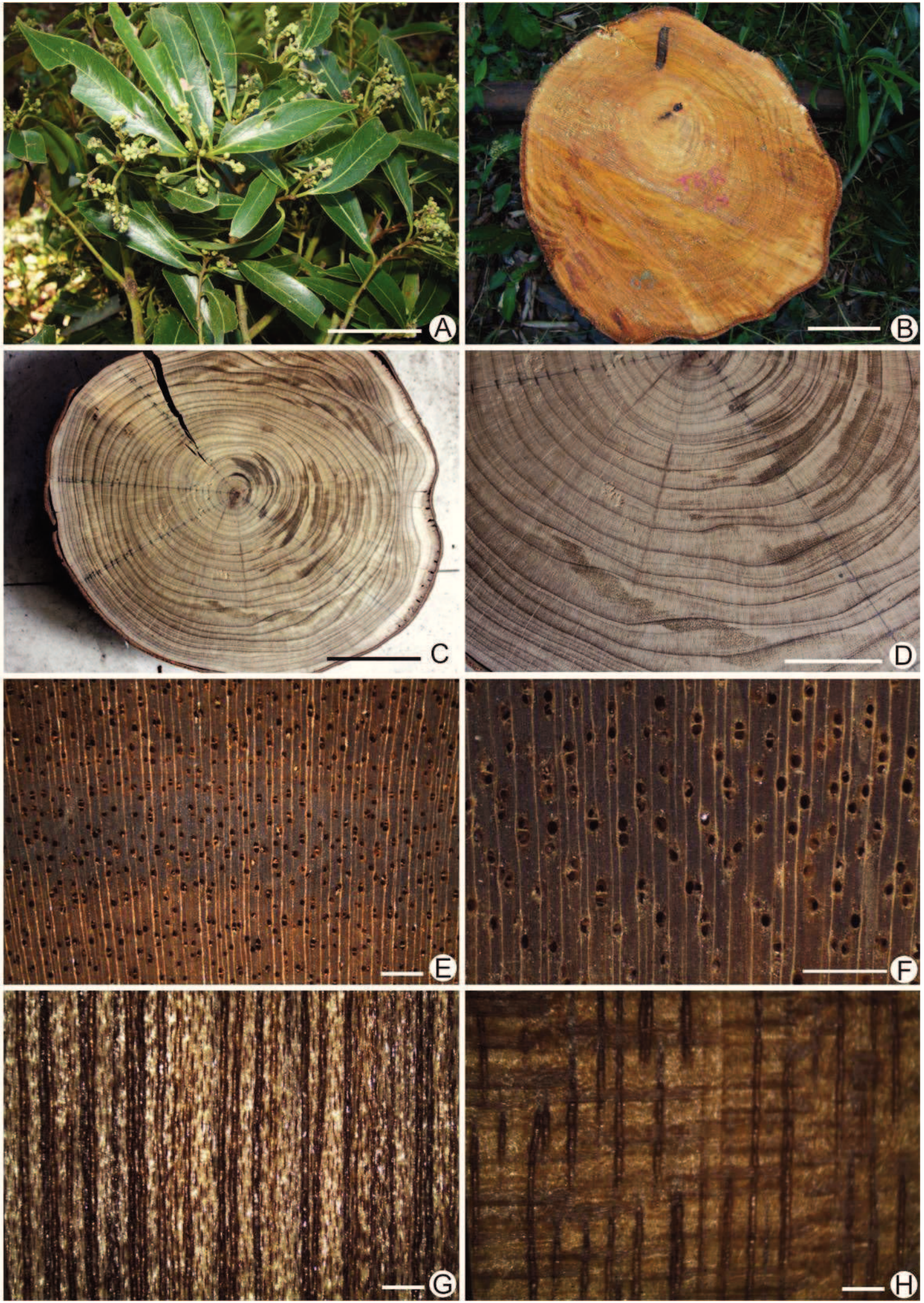
FIGURA 3: Ocotea porosa. A. Vista geral da árvore; B. Disco do tronco recém-cortado; C. Face transversal com anéis de crescimento; D. Secção transversal (10x); E. Secção transversal (20x); F. Secção longitudinal tangencial (10x); G. Secção longitudinal radial (10x). Escalas: A (aprox. 2 m); B (15 cm); C (4 cm); D-G (1 mm).

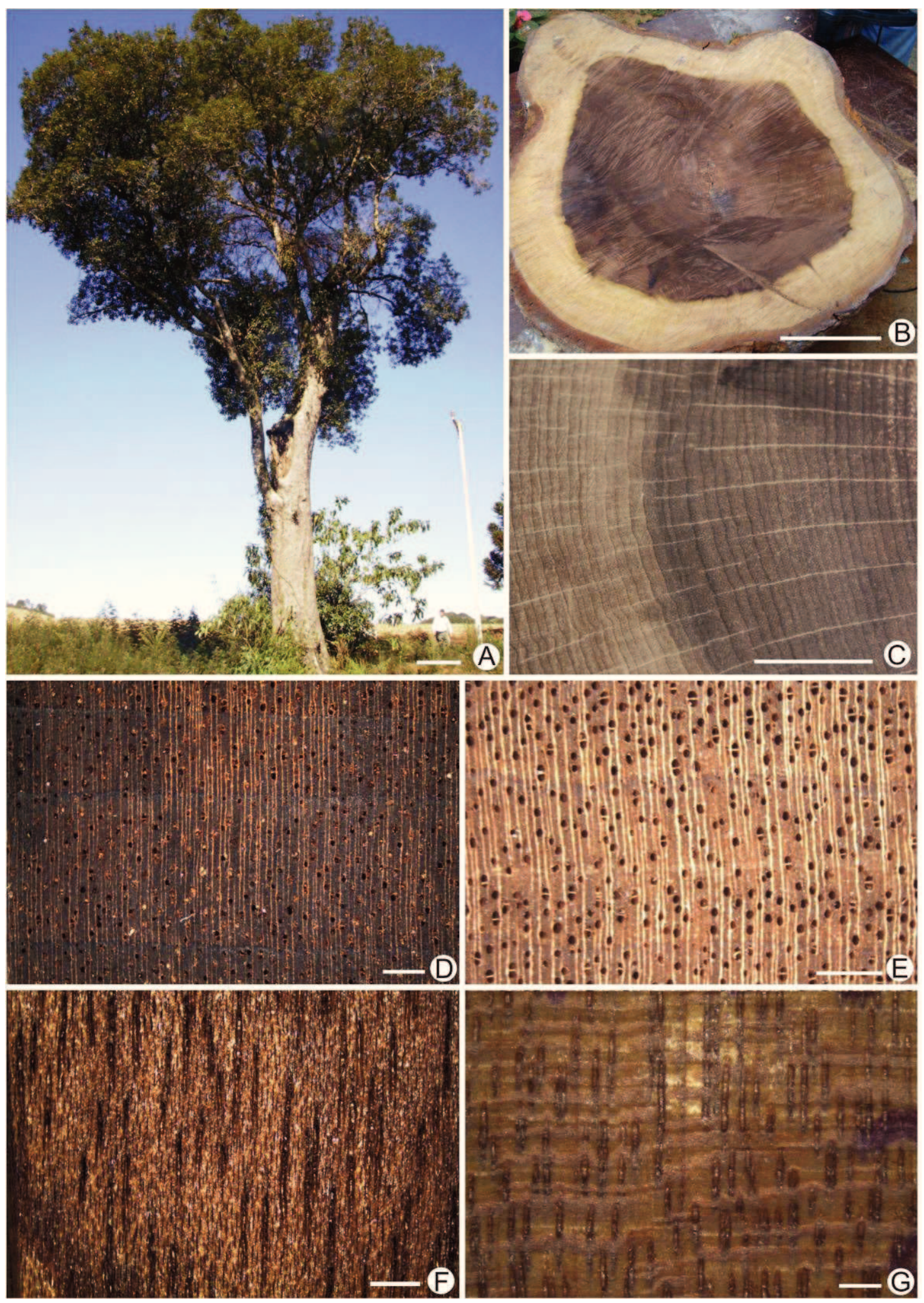


FIGURA 4: Ocotea puberula. A. Ramo da árvore; B. Disco do tronco recém-cortado; C. Disco do tronco lixado; D. Face transversal com anéis de crescimento; E. Secção transversal (10x); F. Secção transversal (20x); G. Secção longitudinal tangencial (10x); H. Secção longitudinal radial (10x). Escalas: A (7 cm); B (6 cm); C (5 cm); D (1 cm); E-H (1 mm).
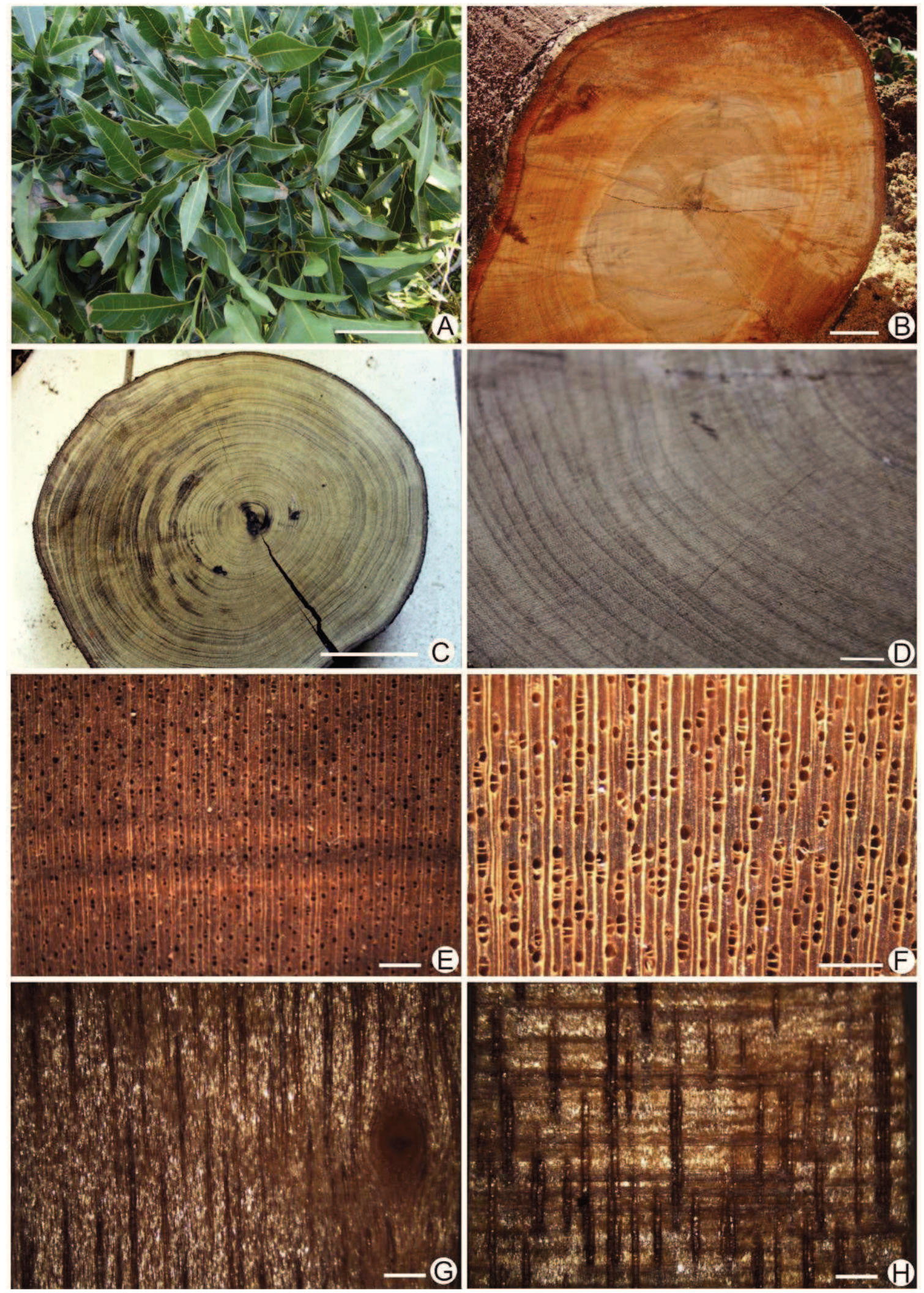
Ocotea pulchella - Canela-lageana (Figura 5A-G)

Características gerais: Cerne/alburno pouco distintos pela cor; cerne marrom-amarelado, com alteração acentuada de cor por foto-oxidação, mudando para amarronzado (Figura 5B); anéis de crescimento distintos, individualizados por zonas fibrosas mais escuras (Figura 5C). Madeira com brilho nas superfícies transversal e longitudinais (Figura 5F-G); com cheiro perceptível, agradável; moderadamente dura ao corte transversal manual; grã direita a revessa; textura média; figura presente em faixas causadas pelas camadas de crescimento (podendo formar "V" ou "U"). Poros: Presentes, visíveis apenas com lente de 10x, pequenos ou médios, de distribuição difusa; frequência média; vasos predominantemente solitários e eventualmente múltiplos radiais com menos de 4 vasos, eventualmente em arranjo diagonal; de formato circular a oval; placas de perfuração não observadas mesmo com lente de 10x; parte dos vasos obstruídos por tilos (Figura 5D-E). Parênquima axial: Não observado, mesmo sob lente de 10x (Figura 5D). Raios: Observados apenas com lente de 10x nas superfícies transversal e longitudinal tangencial (Figura 5F), bem contrastados na superfície radial (Figura 5G), finos, baixos, pouco frequentes. Estruturas estratificadas: Ausentes. Estruturas secretoras: Ausentes. Variantes cambiais: Não observadas. Testes químicos: Teste de Cromazurol negativo.

\section{Ocotea sp. - Canela-loura (Figura 6A-G)}

Características gerais: Cerne/alburno indistintos pela cor; cerne marrom acinzentado (Figura 6B); anéis de crescimento distintos individualizadas por zonas fibrosas mais escuras (Figura 6C); madeira com brilho nas superfícies transversal e longitudinais (Figura 6F-G); sem cheiro perceptível; moderadamente dura ao corte transversal manual; grã direita; textura fina; figura presente em faixas causadas pelas camadas de crescimento (podendo formar "V" ou "U"). Poros: Presentes; visíveis apenas com lente de 10x; pequenos, de distribuição difusa; frequência média; vasos predominantemente solitários e eventualmente múltiplos radiais com menos de 4 vasos, eventualmente em arranjo diagonal; de formato circular a oval; placas de perfuração não observadas mesmo com lente de 10x; parte dos vasos obstruídos por tilos (Figura 6DE). Parênquima axial: Não observado, mesmo sob lente de 10x (Figura 6D). Raios: Observados apenas com lente de 10x na superfície transversal (Figura 6D) e longitudinal tangencial (Figura 6F), bem contrastados na superfície radial (Figura 6G), finos, baixos, pouco frequentes. Estruturas estratificadas: Ausentes. Estruturas secretoras: Ausentes. Variantes cambiais: Não observadas. Testes químicos: Teste de Cromazurol negativo.

Chave dicotômica para diferenciação das espécies pela estrutura macroscópica do lenho

1 - Madeira com odor característico . 2

2 - Odor característico agradável, cerne marromamarelado, parênquima axial indistinto sob lente de $10 \mathrm{x}$ 3

3 - Cerne/alburno distintos pela cor, poros sem arranjo definido Ocotea porosa 3' - Cerne/alburno pouco distintos pela cor, poros eventualmente em arranjo diagonal ....... Ocotea pulchella

2' - Odor característico desagradável, cerne amarelado, parênquima axial distinto sob lente de 10x, escasso ou vasicêntrico Nectandra megapotamica

1' - Madeira sem odor característico . .4 4 - Cerne amarelado, parênquima axial observado sob lente de 10x, paratraqueal escasso ou vasicêntrico Nectandra lanceolata 4' - Cerne branco-amarelado-acinzentado, parênquima axial não observado sob lente de $10 \mathrm{x}$..........5 5 - Poros pequenos, predominantemente solitários, eventualmente dispostos em arranjo diagonal, textura fina .

Ocotea sp.

5' - Poros pequenos ou médios, solitários ou frequentemente geminados ou múltiplos radiais, sem arranjo definido, textura média. Ocotea puberula 
FIGURA 5: Ocotea pulchella. A. Vista geral da árvore; B. Disco do tronco recém-cortado; C. Face transversal com anéis de crescimento; D. Secção transversal (10x); E. Secção transversal (20x); F. Secção longitudinal tangencial (10x); G. Secção longitudinal radial (10x). Escalas: A (aprox. $2 \mathrm{~m})$; B (9 cm); C (2 cm); D-G (1 mm).
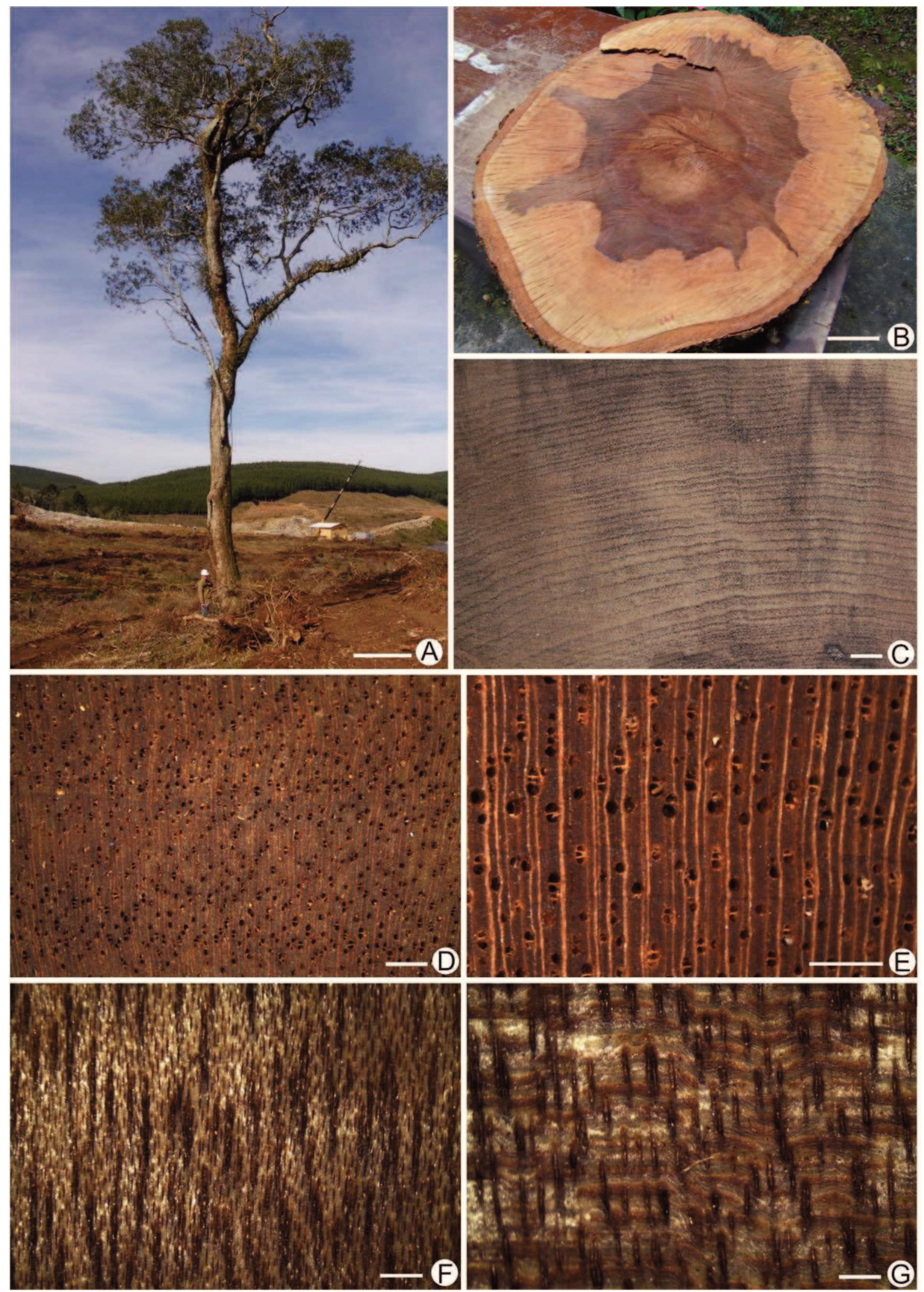
FIGURA 6: Ocotea sp. A. Ramo herborizado; B. Disco do tronco; C. Face transversal com anéis de crescimento; D. Secção transversal (10x); E. Secção transversal (20x); F. Secção longitudinal tangencial (10x); G. Secção longitudinal radial (10x). Escalas: A (4 $\mathrm{cm}) ; \mathrm{B}(1 \mathrm{~cm}) ; \mathrm{C}(0,8 \mathrm{~cm}) ; \mathrm{D}$ a G $(1 \mathrm{~mm})$.
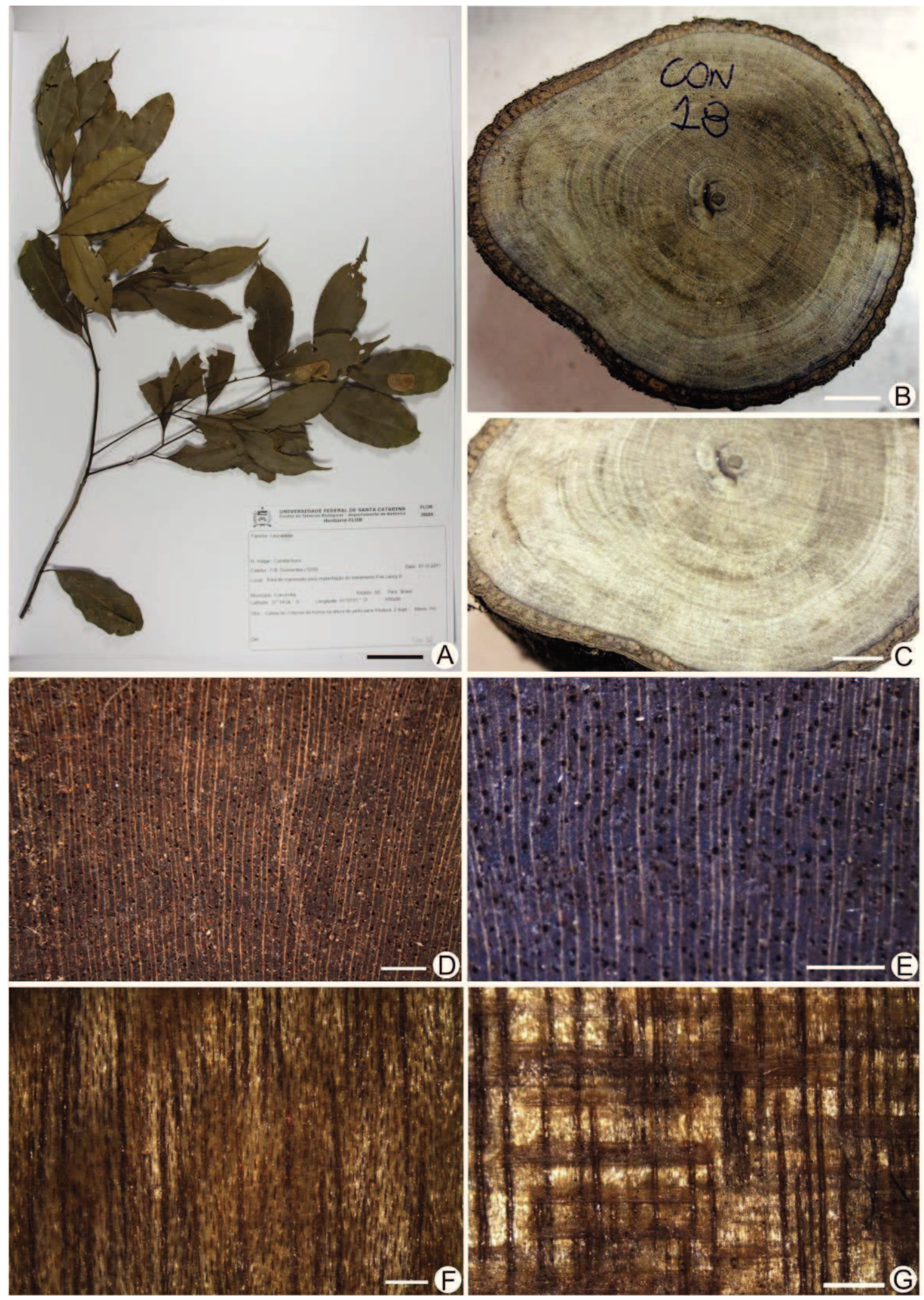


\section{Discussão}

A importância da anatomia do lenho é evidente para a comercialização de madeira, uma vez que a identificação anatômica é indispensável à lisura da transação (ARAÚJO; MATTOS FILHO, 1980). No entanto, o grupo de árvores conhecido popularmente por "canela" representa hoje um grande desafio para os anatomistas quanto se trata de tentar separar as espécies pelas características da madeira. As dificuldades existentes entre os especialistas do meio acadêmico na distinção dos gêneros e das espécies desta família são enormes e, ao longo da cadeia de custódia das madeiras deste grupo, desde os profissionais que elaboram os planos de manejo, passando pelos extratores, transportadores, comerciantes, consumidores e agentes de fiscalização, a identificação, quando realizada, é uníssona: canela. Para os dois maiores laboratórios de identificação do país, quais sejam, Laboratório de Produtos Florestais - LPF, ligado ao Serviço Florestal Brasileiro, em Brasília, e Laboratório de Anatomia de Madeiras, vinculado ao Instituto de Pesquisas Tecnológicas de São Paulo, que recebem um grande fluxo de amostras de madeira para identificação anatômica, oriundas especialmente de apreensões resultantes de ações fiscalizatórias em empresas ligadas ao ramo madeireiro, realizadas por agentes públicos de fiscalização, os laudos de identificação para materiais deste grupo de Lauraceae comumente informam: "Canela - Ocotea/Nectandra". Ainda assim, correm o risco de não estar informando adequadamente sequer o gênero, tendo em vista os recentes rearranjos de espécies que têm sido propostos e os novos gêneros criados em virtude das revisões taxonômicas que têm sido levadas a cabo para os grandes gêneros da família Lauraceae.

Macroscopicamente, as madeiras de canela dos gêneros Ocotea e Nectandra são muito similares, sendo muito difícil a sua distinção, tanto para identificação no nível genérico quanto no nível específico. Entre os caracteres anatômicos macroscópicos e organolépticos do lenho que puderam ser considerados na tentativa de diferenciação das espécies analisadas no presente trabalho estão: cor, odor, disposição dos elementos de vaso e parênquima axial.
A coloração amarelada ou amarronzada, com nítida foto-oxidação durante a secagem, e o brilho perceptível nas faces longitudinais do lenho parecem ser comuns neste grupo. Observou-se que as madeiras das espécies de Nectandra analisadas tendem mais para a cor amarela, especialmente quando recém-cortadas, e escurecem pouco, tornando-se amarronzadas após secas e difíceis de serem distintas entre si, considerando-se apenas este caráter. Já as espécies de $O$. porosa e $O$. pulchella apresentaram cerne marrom desde o momento do corte e, quando secas, a coloração acentuou-se e escureceu, adquirindo, a primeira, um tom mais oliváceo. Ocotea puberula se apresentou mais clara, esbranquiçada, no momento do corte, escurecendo para marrom acinzentado após a secagem, muito similar à cor de Ocotea sp., o que torna inviável a distinção destas últimas pela cor. Na literatura, as espécies de Ocotea e Nectandra não possuem grande variação na cor, sendo descritas como madeiras amareladas, castanho-amareladas, marrom-amareladas, amareloesverdeadas, amarelo-oliváceas, marrom-douradas ou amarelo-grisáceo (KRIBS, 1959; LOUREIRO; SILVA, 1968; VAR DER SLOOTEN et al., 1970; DECHAMPS, 1979; MAINIERI, 1983). Apesar da subjetividade que a observação da cor do lenho possa fornecer ao nível macroscópico, este caráter tem sido utilizado em chaves dicotômicas para identificação anatômica de madeiras, como em Mainieri (1983), Coradin et al. (1991), Zenid e Ceccantini (2007), Coradin et al. (2009), inclusive como elemento principal de entrada na chave, como em Camargos (1996).

A presença de odores característicos, agradáveis ou desagradáveis no lenho, como o observado para $O$. porosa, O. pulchella e N. megapotamica, é comum ao grupo das canelas e foi, possivelmente, um dos caracteres mais importantes para separação das espécies. Esses odores são amplamente relatados inclusive nos nomes populares, por vezes chulos, para espécies deste grupo, como: canela-fedorenta, canela-fedida, canelabosta, canela-merda, canela-cheirosa, canela-de-cheiro, canela-de-mau-cheiro, canela-cânfora, casca-cheirosa, catinga-de-negro, louro-cânfora, louro-cheiroso, paucravo, entre muitos outros (CAMARGOS et al., 2001). Ochioni e Mattos Filho (1947) mencionam o cheiro de 
“funcho" para O. fragrantissima. Vattimo (1956) indica a presença de aroma de anis para $O$. puberula e cheiro "nauseabundo" para O. spectabilis. Mainieri (1971) indica cheiro ativo e agradável para $O$. cymbarum e a ausência de cheiro para Sextonia rubra. Loureiro e Silva (1968) indicam cheiro "aromático ativo" para Ocotea cymbarum. Kribs (1959) descreve cheiro agradável para O. schomburgkiana, O. floribunda, O. glomerata, resinoso-agradável para $O$. cymbarum, Nectandra globosa, N. sanguinea e N. turbacensis, odor desagradável para $O$. bullata e odor de cânfora para O. usambarensis. Rizzini (1971) destaca o cheiro característico, resinoso e agradável de $O$. porosa, resinoso de $O$. catharinensis, odor de sassafrás e odor de canela para as variedades fisiológicas de $O$. odorifera. Usa o termo "perfumada" para Sextonia rubra, "aromática" para O. aciphylla, "fétida" para $N$. cissiflora (ali descrita como N. myriantha). Indica odor desagradável para $N$. megapotamica e não indica a presença de odores para $N$. puberula, $N$. reticulata e O. pulchella. Castiglioni (1962) refere-se ao odor de N. megapotamica como desagradável e penetrante, ao de $N$. angustifolia como pronunciado e agradável, $N$. lanceolata e N. cuspidata com odores pouco pronunciados. Rohwer (1986), diante da dificuldade de diferenciar espécimes de herbário de $O$. porosa e $O$. catharinensis, recebeu de Richter comunicação pessoal indicando que o cheiro de O. porosa é "perfumado" e de $O$. catharinensis quase insuportavelmente fétido.

A disposição dos vasos arranjados diagonalmente, como observado no lenho das árvores de $N$. lanceolata, $N$. megapotamica, O. pulchella e Ocotea sp. apresentou grande variação entre as amostras analisadas, e eventualmente dentro das mesmas amostras. Assim, não deve ser considerado um caráter diagnóstico forte, como se observa em Sextonia rubra (KRIBS, 1959; CORADIN et al. 2009), apesar de não ter sido relatado por Dechamps (1979) e Feldato et al. (1989).

A distinção macroscópica do parênquima axial no lenho das espécies de Ocotea e Nectandra estudadas também se mostrou uma tarefa árdua e questionável. Macroscopicamente, com auxílio de lupa de 10x, é muito difícil distinguir a presença de parênquima axial e se este é paratraqueal escasso ou vasicêntrico, sobretudo se for vasicêntrico pouco abundante ou delgado, especialmente em razão da presença de células oleíferas/mucilaginosas vinculadas ao parênquima axial, o que leva a uma indefinição sobre a real abrangência deste em torno dos poros. No presente estudo, pôde-se observar, sob lente de 10x, parênquima axial paratraqueal escasso ou vasicêntrico em $N$. lanceolata e $N$. megapotamica. Nas demais espécies, sob esta ótica, o parênquima axial não se mostrou distinto. Kribs (1959) também indica como indistinto sob lente de 10x o parênquima axial de Ocotea cymbarum, O. bullata, O. glomerata, O. schomburgkiana, O. floribunda, O. usambarensis, Nectandra globosa, N. sanguinea e $N$. turbacensis. Loureiro e Silva (1968) descrevem como praticamente indistinto, mesmo com auxílio de lentes, o parênquima axial de O. cymbarum. A chave de identificação de madeiras do IPT, elaborada por Zenid e Ceccantini (2007) consideram todas as espécies de Ocotea e Nectandra com parênquima axial indistinto, mesmo sob lente.

Não foram observadas dissimilaridades quanto aos raios nas faces radial e tangencial entre as espécies avaliadas macroscopicamente. Bem como também não foram observadas gomas. A presença de gomas foi relatada apenas por León (1999-2000) em O. aciphylla e $O$. catharinensis. Os tilos, eventualmente presentes nas amostras avaliadas, não se mostraram um caráter significativo para a diferenciação das espécies. Não houve reação no teste do Cromazurol-S em nenhuma das espécies avaliadas.

Concluímos então que os lenhos das árvores das espécies de Ocotea e Nectandra ora analisados, bem como os dados disponíveis na literatura, apresentam alto grau de similaridade. A análise macroscópica de caracteres anatômicos por si só do lenho de espécies de Ocotea e Nectandra não permite a clara distinção das espécies. Entretanto, caracteres organolépticos, especialmente cor e odores, devem ser usados em conjunto com os caracteres anatômicos macroscópicos, para tornar possível a distinção. Ainda assim, há certo grau de subjetividade nesses caracteres, que podem facilmente levar ao cometimento de erros. 


\section{Agradecimentos}

Ao IBAMA pela concessão de afastamento para capacitação (processo 02026.000114/2010-77). Pelas contribuições logísticas e técnicas ao longo da pesquisa, agradecemos: Dr. João Baitello; Dr. Leandro Cézanne de Souza Assis; Dra. Vera Rauber Coradin (LPF/SFB), Profa. Dirlei Inês Thiel; Dr. Hans Georg Richter; Billie \& Marcie Miller; A. Bermudez (ESALQ/USP) e Dr. Rafael Trevisan (FLOR/BOT/UFSC).

\section{Referências}

ARAÚJO, P. A. M.; MATTOS FILHO, A. de. A importância da anatomia do lenho para a comercialização da madeira. Rodriguésia, Rio de Janeiro, v. 32, n. 53, p. 315-318, 1980.

CALLADO, C. H.; COSTA, C. G. Wood anatomy of some Anaueria and Beilschmiedia species (Lauraceae). IAWA Journal, Leiden, v. 18, n. 3, p. 247-259, 1997.

CAMARGOS, J. A. A. Madeiras comerciais de Mato Grosso: chave de identificação. Brasília: IBAMA, 1996. 82 p.

CAMARGOS, J. A. A.; CORADIN, V. T. R.; CZARNESKI, C. M.; OLIVEIRA, D.; MEGUERDITCHIAN, I. Catálogo de árvores do Brasil. Brasília: IBAMA, 2001. 896 p.

CASTIGLIONI, J. A. El leño secondario de las especies argentinas de Nectandra. Revista de Investigaciones Forestales, Buenos Aires, v. 3, n. 1, p. 1-15, 1962.

CORADIN, V. T. R.; CAMARGOS, J. A. A.; PASTORE, T. C. M.; CHRISTO, A. G. Madeiras comerciais do Brasil: chave interativa de identificação baseada em caracteres macroscópicos. Versão 1.0. 2009.

CORADIN, V. T. R.; MARQUES, M. H. B.; CAMARGOS, J. A. A.; MUÑIZ, G. B. Chave de identificação das principais madeiras da Floresta Nacional do Tapajós. Brasília: IBAMA, 1991. 51 p.

CORADIN, V. T. R.; MUÑIZ, G. M. B. Normas e procedimentos em estudos de anatomia de madeira: I. Angiospermae. II. Gimnospermae. Vol. 15. Brasília: LPF, 1991. 19 p.

DECHAMPS, R. Etude anatomique de bois d'Amérique du Sud. I. Acanthaceae à Lecythidaceae. Annales Musée Royal de l'Afrique Centrale, Tervuren. Sér. IN-8, Sci. econ, n. 10, 1979. 332 p.

FEDAlTO, L. C.; MENDES, I. C. A.; CORADIN, V. T. R. Madeiras da Amazônia: descrição do lenho de 40 espécies ocorrentes na Floresta Nacional do Tapajós. Brasília: IBAMA, 1989. $156 \mathrm{p}$.

KOSTERMANS, A. J. G. H. Lauraceae. Reinwardtia, Bogor, v. 4, n. 2, p. 193-256, 1957.

KRIBS, D. A. Commercial foreign woods on the American market. Pennsylvania: Pennsylvania State University, 1959. 241 p.

KUKACHKA, B. F.; MILLER, R. B. A chemical spot-test for aluminum and its value in wood identification. IAWA Bulletin, Leiden, v. 1, p. 104-109, 1980.

LEÓN, W. J. Anatomía del leño de 17 especies del género Ocotea
Aublet (Lauraceae). Pittieria, Mérida, v. 1, n. 29-30, p. 53-65, 1999-2000.

LEÓN, W. J.; ESPINOZA DE PERNÍA, N. Estudio anatômico del leño de ocho especies del género Aniba Aublet (Lauraceae). Revista Forestal Venezolana, Mérida, v. 44. n. 1, p. 37-46, 2000a. LEÓN, W. J.; ESPINOZA DE PERNÍA, N. Estudio anatômico del leño de siete especies del género Beilschmiedia Nees (Lauraceae). Revista Forestal Venezolana, Mérida, v. 44, n. 1, p. 47-56, 2000 b. LOUREIRO, A. A.; SILVA, M. F. da. Catálogo das madeiras da Amazônia. Vol. 1. Belém: SUDAM, 1968. 433 p.

MAINIERI, C. 25 madeiras da Amazônia de valor comercial, caracterização macroscópica, usos comuns e índices qualificativos. São Paulo: IPT, 1971. 33 p.

MAINIERI, C. Manual de identificação das principais madeiras comerciais brasileiras. São Paulo: IPT, 1983. 241 p.

METCALFE, C.; CHALK, L. Anatomy of the Dicotyledons. Vol. II. 2. ed. Oxford: Claredon Press, 1985. 297 p.

OCHIONI, P.; MATTOS FILHO, A. Estudo anatômico do lenho secundário do puchury-rana Ocotea fragrantissima Ducke. Rodriguésia, Rio de janeiro, v. 10, n. 21, p. 1-12, 1947.

OLIVEIRA, C. W. de; CALlADO, C. H.; MARQUETE, O. Anatomia do lenho de espécies do gênero Nectandra Rol. ex Rottb. (Lauraceae). Rodriguésia, Rio de Janeiro, v. 52, n. 81, p. 125-134, 2001.

RICHTER, H. G. Anatomie des sekundären xylems und der rinde der Lauraceae. Sonderbände des Naturwiss Vereins, Hamburg, n. 5, p. 1-148, 1981.

RICHTER, H. G. Mature secondary xylem. In: METCALFE, C. R. (Ed.). Anatomy of the Dicotyledons. 2. ed. Oxford: Claredon Press, 1987. p. 162-171.

RICHTER, H. G. Wood and bark anatomy of Lauraceae. III: Aspidostemon Rohwer \& Richter. IAWA Bulletin, Leiden, n. 11, p. 47-56, 1990.

RIZZINI, C. T. Plantas do Brasil. Árvores e madeiras úteis do Brasil: manual de dendrologia brasileira. São Paulo: Edgar Blücher/ EDUSP, 1971. 294 p.

ROHWER, J. G. Prodromus einer monographie der gattung Ocotea Aubl. (Lauraceae) sensu lato. Hamburg: Hubert \& Co, 1986. $278 \mathrm{p}$.

ROHWER, J. G. Borderline cases between Ocotea, Nectandra and Phoebe (Lauraceae): the "marginal" species of the Ocotea helicterifolia-group including the Ocotea heydeana-group. Botanische Jahrbücher fur Systematik, Pflanzengeschichte und Pflanzengeographie. Leipzig, n. 112, p. 365-397, 1991.

ROHWER, J. G. Lauraceae. In: KUBITZKI, K.; ROHWER, J. G.; BITTRICH, V. (Ed.). The families and genera of vascular plants II. Berlin: Springer-Verlag, 1993. p. 366-391.

SOLEREDER, H. Laurinae. In: SOLEREDER, H.; BOODLE, L. A.; FRITSCH, F. E. (Ed.). Systematic anatomy of the dicotyledons: a handbook for laboratories of pure and applied botany. Vol. II. Oxford: Clarendo Press. 1908. p. 702-706.

STERN, W. L. Comparative anatomy of xylem and phylogeny of Lauraceae. Tropical Woods, New Haven, n. 100, p. 1-75, 1954.

TUPPER, W. W. A comparative study of Lauraceous woods. American Journal of Botany, St Louis, v. 14, p. 520-525, 1927. 
VAN DER SLOOTEN, H. J.; ACOSTA-CONTRERAS. I.; AAS, P. S. Madeiras latinoamericanas. IV. Nectandra sp., Ocotea austinii, Persea sp. aff. vesticula, Persea schiedeana. Turrialba, San José, v. 20, n. 2, p. 223-232, 1970.

VATTIMO, I. de. O gênero Ocotea Aubl. no sul do Brasil I. Espécies de Santa Catarina e do Paraná. Rodriguésia, Rio de Janeiro, v. 1819, n. 30-31, p. 265-350, 1956.

ZENID, G. J.; CECCANTINI, G. C. T. Identificação macroscópica de madeiras. São Paulo: IPT, 2007. 51 p. 